LETTER TO THE EDITOR

\section{The use of an adequate mathematical model is crucial to evaluate vaccine effectiveness}

\author{
Jorge A Gomez ${ }^{+}$, Javier Nieto ${ }^{1}$, \\ Maria Rangel, Eduardo Ortega-Barria
}

\author{
GlaxoSmithKline Biologicals, \\ Latin America and the Caribbean, Buenos Aires, Argentina
}

A recent paper by El Khoury et al. (2011) reported estimates of effectiveness of the RotaTeq ${ }^{\circledR}$ vaccine against rotavirus-related hospitalisations in Brazil of $93 \%$. After carefully examination of the data presented in this paper, it appears that there are important limitations on this study leading to a potential overestimation of the reported vaccine effectiveness. Although the analysis applies a model validated by Rose and Singer in 2008, the conclusions in the Khoury's paper are beyond the original model capabilities. The model used was developed to project expected rotavirus vaccine efficacy in settings where applying unadjusted efficacy data could overestimate the benefits of immunisation. To generate a weighted efficacy estimate for Brazil, El Khoury et al. (2011) used serotype-specific vaccine efficacy estimates against hospitalised rotavirus gastroenteritis (RGE) from the Rotavirus Efficacy and Safety Trial (REST) (Vesikari et al. 2006) and the distribution of rotavirus types observed in four cities of Brazil (Goiânia, Porto Alegre, Salvador and São Paulo) from 2005-2006. It is difficult to determine whether the strain-specific vaccine efficacy against hospitalised RGE observed in the REST study would be obtained if that study were performed in Brazil. Indeed, differences in access to health care services and differences in health seeking behaviour between locations in Brazil may alter the disease severity presentation of hospitalised children with rotavirus diarrhoea and therefore impact the estimated vaccine efficacy. Thus, it is a daring assumption to conclude that the "true" rotavirus strain distribution in Brazil is equivalent to a weighted average of the strain distributions observed in the four cities studied given the known dynamics of rotavirus strain circulation profile in Brazil (Leite et al. 2008).

El Khoury et al. (2011) acknowledged that although the model utilized was developed to estimate projected efficacy, they used the term "projected effectiveness" throughout the paper because their objective was to proj-

+ Corresponding author: jorge.a.gomez@gsk.com

Received 29 February 2012

Accepted 28 March 2012 ect the real-life impact of RotaTeq ${ }^{\circledR}$ vaccine on the reduction of rotavirus-related hospitalisations. This is an important shortcoming of the El Khoury el al. (2011) paper since the two terms are not equivalent and therefore the reported projected effectiveness estimate for Brazil is incorrect. It is well known that rotavirus vaccine effectiveness in a given population is not related to the virus strain type coverage only. Other factors such as malnutrition, transplacental maternal and breast milk antibodies and co-infecting pathogens could interfere with an infants' immune response to the vaccine. Indeed, other live oral vaccines against typhoid, cholera, polio as well as earlier rotavirus vaccines have historically performed less well than expected in developing countries (John 1976, Patriarca et al. 1991, Su-Arehawaratana et al. 1992, Levine 1997, Glass et al. 2006).

The per protocol analysis and efficacy subset of the REST study contained only $8 \%$ of the participants from Latin American/Caribbean countries and Puerto Rico and reported an efficacy against rotavirus hospitalisations in these countries of 50.2 [95\% confidence interval (CI) $<0.0-99.1 \%]$, based on a very limited number of cases (Vesikari et al. 2007). The model inputs for baseline serotype-specific vaccine efficacy estimates were derived from the REST type-specific results, using a combined efficacy against RGE-related hospitalisations and emergency department visits due to each serotype (Vesikari et al. 2006), but the study makes projections on the vaccine effectiveness against RGE-related hospitalisations. Therefore the model used by El Khoury et al. (2011) only estimate adjusted vaccine efficacy of Rotateq ${ }^{\circledR}$ and not real-life effectiveness of Rotateq ${ }^{\circledR}$ for Brazil.

In addition to the aforementioned shortcomings, the estimated results from El Khoury et al. (2011) are significantly different to the effectiveness observed for the same vaccine in a recent Phase IV study conducted in Latin America (Patel et al. 2009). The observed reallife vaccine effectiveness for the complete schedule of RotaTeq $^{\circledR}$ against rotavirus disease requiring hospital admission or treatment with intravenous hydration in Nicaragua was $46 \%$ (95\% CI, $18-64 \%)$ in the Patel et al. (2009) study.

In summary, we agree the estimated type-adjusted vaccine efficacy of RotaTeq ${ }^{\circledR}$ vaccine $(93 \%)$ may be closer to the real life value for Brazil, than the original value observed in the REST study for Latin American countries (50.2\%; 95\% CI: < 0-99.1\%) (Vesikari et al. 2007). However, it is important to highlight that the model utilized by El Khoury et al. (2011) was not intended to estimate real-life vaccine effectiveness, but rather efficacy estimates extrapolated from data obtained in a very controlled setting (clinical randomized controlled trial) covering mainly study sites located outside Latin America. When mathematical models are used, it is always valuable to clearly understand the goals the model was developed for. Therefore, from our point of view, the reported vaccine effectiveness for Brazil is overestimated based on the incorrect use of a validated model, assumptions not supported by scientific evidence and a significant simplification of rotavirus epidemiology in Brazil. 


\section{REFERENCES}

El Khoury AC, Mast TC, Ciarlet M, Markson L, Goveia MG, Munford V, Rácz ML 2011. Projecting the effectiveness of RotaTeq ${ }^{\circledR}$ against rotavirus-related hospitalisations in Brazil. Mem Inst Oswaldo Cruz 106: 541-545.

Glass RI, Parashar UD, Bresee JS, Turcios R, Fischer TK, Widdowson MA, Jiang B, Gentsch JR 2006. Rotavirus vaccines: current prospects and future challenges. Lancet 368: 323-332.

John TJ 1976. Antibody response of infants in tropics to five doses of oral polio vaccine. $\mathrm{Br}$ Med $J$ 1: 812.

Leite JPG, Carvalho-Costa FA, Linhares AC 2008. Group A rotavirus genotypes and the ongoing Brazilian experience - A Review. Mem Inst Oswaldo Cruz 103: 745-753.

Levine MM 1997. Oral vaccines against cholera: lessons from Vietnam and elsewhere. Lancet 349: 220-221.

Patel M, Pedreira C, De Oliveira LH 2009. Association between pentavalent rotavirus vaccine and severe rotavirus diarrhoea among children in Nicaragua. JAMA 301: 2243-2251.

Patriarca PA, Wright PF, John TJ 1991. Factors affecting the immunogenicity of oral poliovirus vaccine in developing countries: review. Rev Infect Dis 13: 926-939.

Rose J, Singer ME 2008. Projecting vaccine efficacy: accounting for geographic strain variations. Pharmacoeconomics 26: 185-189.

Su-Arehawaratana P, Singharaj P, Taylor DN, Hoge C, Trofa A, Kuvanont K, Migasena S, Pitisuttitham P, Lim YL, Losonsky G 1992. Safety and immunogenicity of different immunization regimens of CVD 103-HgR live oral cholera vaccine in soldiers and civilians in Thailand. $J$ Infect Dis 165: 1042-1048.

Vesikari T, Itzler R, Matson DO 2007. Efficacy of a pentavalent rotavirus vaccine in reducing rotavirus-associated health care utilization across three regions (11 countries). Int $J$ Infect Dis 11 (Suppl 2): S29-S35.

Vesikari T, Matson DO, Dennehy P 2006. Safety and efficacy of a pentavalent human-bovine (WC3) reassortant rotavirus vaccine. N Engl J Med 354: 23-33.

\section{REPLY}

Comments in regards to Jorge A Gomez et al.'s letter

Gomez et al. agree that the rotavirus serotype-adjusted vaccine efficacy of RotaTeq ${ }^{\circledR}$, as projected in our paper, of $93 \%$ (El Khoury et al. 2011) may be closer to the reallife effectiveness against severe disease in Brazil than the original efficacy estimate observed in the large-scale study [Rotavirus Efficacy and Safety Trial (REST)] for countries in Latin America (Vesikari et al. 2006). However, Gomez et al. raise some concerns about the limitations of our modelling and the use of the term "projected effectiveness" as opposed to "extrapolated efficacy".

Their main concerns regarding the use of the efficacy results from REST to project the vaccine effectiveness in Brazil have been addressed in the discussion section of our paper (El Khoury et al. 2011). We state that changes in rotavirus patterns over time and the populations evaluated may have an impact on vaccine effectiveness. Because rotavirus serotype prevalence can change from region to region and year to year, we used recently available rotavirus serotype prevalence data from Brazil to conduct the projections.

We also highlight that the modelled effectiveness of RotaTeq $^{\circledR}$ in Brazil falls between the published estimates of effectiveness in Nicaragua within one year after vaccination (69\%) (Patel et al. 2009) and the data from the United States (US) showing that RotaTeq ${ }^{\circledR}$ prevented $100 \%$ of rotavirus gastroenteritis-related hospitalizations and emergency department visits (Wang et al. 2010). As Gomez et al. point out, rotavirus vaccine effectiveness does vary by region where estimates positively correlate with improved childhood health indicators (Fischer-Walker \& Black 2011). Thus, RotaTeq ${ }^{\circledR}$ is likely to have a vaccine effectiveness in Brazil at estimates closer to what was observed in the US than those observed in Nicaragua, which would be our estimate of $93 \%$. In addition, local health care utilization patterns and policies can strongly affect how children are hospitalized and, while the Patel et al. (2009) study cited by Gomez et al. showed $49 \%$ vaccine effectiveness for hospitalization and Phase IV therapy, the same study showed $77 \%$ vaccine effectiveness for very severe disease using the Vesikari scale $>=15$ (Patel et al. 2009). In addition, the Merck-Nicaragua Ministry of Health collaborative study showed vaccine effectiveness during two years of follow-up against severe disease (Vesikari >= 11) in children receiving three doses of RotaTeq ${ }^{\circledR}$ of $76 \%$ (95\% confidence interval; 63-84\%) (Mast et al. 2011). So, if in Brazil, hospitalized admissions for rotavirus tend to be more severe than Nicaragua, then the Nicaragua data could predict approximately $76-77 \%$ vaccine effectiveness for hospitalization in Brazil and this narrows further the range of estimates around our projected $93 \%$.

Regarding the use of the term "projected effectiveness", as opposed to "extrapolated efficacy", although we acknowledge the confusion around the use of this terminology in the literature, we decided to use the term "projected effectiveness" because we were not only extrapolating the efficacy from REST using the rotavirus serotype prevalence in Brazil, but also making assumptions about the efficacy of untypeable rotavirus serotypes and vaccine coverage rates in a routine setting. In addition, efficacy projections in other disease areas often refer to extrapolations that go beyond the length of the trial for the same population studied in the trial, something that was not the focus of our paper. Vaccine effectiveness analyses often aim to understand clinical trial results as applied to broader populations not specifically included in the trial, which was our objective in this paper. Therefore, we decided that the term "effectiveness" was a better reflection of our modelling approach than "extrapolated efficacy". We acknowledge that some factors in real-world settings could potentially impact outcomes, but we aimed to address as many as possible, including a number of non-trial parameters, such as vaccine coverage rates, hospitalization rates for rotavirus in Brazil (Satori et al. 2008), rotavirus serotype prevalence in Brazil (Munford et al. 2009) and assumptions related to the efficacy of the vaccine against untypeable serotypes.

In conclusion, as in any other mathematical model, the validity of the results depends on the assumptions used in the analyses. In the absence of real-world data, our modelling predicts a substantial impact of RotaTeq ${ }^{\circledR}$ against rotavirus-related hospitalizations in Brazil. These data will help policy makers assess the benefits of vaccination with the pentavalent rotavirus vaccine. 


\section{REFERENCES}

El Khoury AC, Mast TC, Ciarlet M, Markson L, Goveia MG, Munford V, Rácz ML 2011. Projecting the effectiveness of RotaTeq ${ }^{\circledR}$ against rotavirus-related hospitalisations in Brazil. Mem Inst Oswaldo Cruz 106: 541-545.

Fisher-Walker C, Black R 2011. Rotavirus vaccine and diarrhea mortality: quantifying regional variation in effect size. BMC Public Health 11 (Suppl. 3): S16.

Mast TC, Khawaja S, Espinoza F 2011. Case-control study of effectiveness of vaccination with pentavalent rotavirus vaccine in $\mathrm{Ni}$ caragua. Pediatr Infect Dis J 30: e209-e215.

Munford V, Gilio AE, de Souza EC, Cardoso DM, Cardoso DD, Borges AM, Costa PS, Melgaço IA, Rosa H, Carvalho PR, Goldani MZ, Moreira ED Jr, Santana C, El Khoury A, Ikedo F, Rácz ML 2009. Rotavirus gastroenteritis in children in 4 regions in Brazil: a hospital-based surveillance study. J Infect Dis 200 (Suppl. 1): S106-S113.

Patel M, Pedreira C, De Oliveira LH 2009. Association between pentavalent rotavirus vaccine and severe rotavirus diarrhoea among children in Nicaragua. JAMA 301: 2243-2251.

Satori AM, Valentim J, de Soarez PC, Novaes HM 2008. Rotavirus morbidity and mortality in children in Brazil. Rev Panam Salud Publica 23: 92-100.
Vesikari T, Matson DO, Dennehy P 2006. Safety and efficacy of a pentavalent human-bovine (WC3) reassortant rotavirus vaccine. N Engl J Med 354: 23-33.

Wang FT, Mast TC, Glass R 2010. Effectiveness of the pentavalent rotavirus vaccine in preventing gastroenteritis in the United States. Pediatrics 125: e208-e213.

Antoine Chaouki El Khoury Leona Markson Global Health Outcomes, Merck \& Company, Inc, West Point, PA, USA

Thomas Christopher Mast Merck Research Laboratories, Merck \& Company, Inc, Upper Gwynedd, PA, USA

Max Ciarlet Clinical Research and Development, Novartis Vaccines \& Diagnostics, Cambridge, MA, USA

Michelle Gail Goveia Global Medical Affairs, Merck \& Company, Inc, West Point, PA, USA

Veridiana Munford

Maria Lucia Ràcz

Departamento de Microbiologia, Instituto de Ciências Biomédicas, Universidade de São Paulo, São Paulo, SP, Brasil 